\title{
Dichotomy in the population of young AGN: optical, radio and X-ray properties
}

\author{
Magdalena Kunert-Bajraszewska ${ }^{1, \star}$ \\ Toruń Centre for Astronomy, Faculty of Physics, Astronomy and Informatics, NCU, Grudziadzka 5, 87-100 Toruń, Poland \\ Received XXXX, accepted XXXX \\ Published online XXXX
}

Key words galaxies: active - galaxies: evolution - radio continuum: galaxies

There are numerous examples of radio sources with various sizes which surprisingly exhibit very similar morphology. This observational fact helped to create a standard evolutionary model in which young and small radio-loud active galactic nuclei (AGN) called gigahertz-peaked spectrum (GPS) sources and compact steep spectrum (CSS) sources, become largescale radio objects. However, many details of this evolutionary process are still unclear. We explored evolution scenarios of radio-loud AGN using new radio, optical and X-ray data of so far unstudied low luminosity compact (LLC) sources and we summarize the results in this paper. Our studies show that the evolutionary track is very 'personalized' although we can mention common factors affecting it. These are interaction with the ambient medium and AGN power. The second feature affects the production of the radio jets which if they are weak are more vulnerable for instabilities and disruption. Thus not all GPS and CSS sources will be able to develop large scale morphologies. Many will fade away being middle-aged ( $10^{5}$ years). It seems that only radio strong, high excitation compact AGN can be progenitors of large-scale FR II radio sources.

Copyright line will be provided by the publisher

\section{Introduction}

Evolutionary paths of radio sources are commonly plotted in a radio power - linear size plane. This parametrization of the radio source behaviour does serve to capture the major evolutionary trends that can be compared with observations since it involves the two most evident, easily measured features of extended radio sources. In the general scenario of the evolution of powerful radio-loud AGN (Readhead et al. 1996; Fanti et al. 1995; O'Dea \& Baum 1997), the younger and smaller gigahertz-peaked spectrum (GPS) and compact steep spectrum (CSS) sources become large scale FR I and FR II objects (Fanaroff \& Riley, 1974).

However, the growing number of observations of low power radio sources and results of their analysis suggest that not one but at least two evolutionary paths exist (Snellen et al. 2000; Marecki et al. 2003; Kunert-Bajraszewska et al. 2010; An \& Baan 2012; Maciel \& Aleksander 2014; Turner \& Shabala 2015). The determining factors for the evolution of radio objects can be related either to jet-interstellar medium (ISM) interactions or central engine (differences in the accretion mode or black hole spin, instabilities in the accretion flow). During their evolution the radio jets start to cross the ISM and try to leave the host galaxy. The interaction with the ISM can be very strong in GPS and CSS sources and it seems to be a crucial point in the evolution of radio sources (Labiano 2008; Holt, Tadhunter \& Morganti 2009; Kawakatu et al. 2009; Perucho, Quilis \& Martí 2011;

^ Corresponding author: e-mail: magda@astro.uni.torun.pl
Wagner et al. 2012; Chandola, Gupta \& Saikia 2013; Dallacasa et al. 2013; Maccagni et al. 2014; Geréb et al. 2015).

On the other hand the study of the optical properties of radio-loud AGN suggests the existence of two radio source populations where the main discriminant is the accretion rate onto the central black hole (Hardcastle, Evans \& Croston 2007; Buttiglione et al. 2010; Kunert-Bajraszewska \& Labiano 2010; Best \& Heckman 2012). According to these studies high excitation galaxies (HEG) are distinctive in strong evolution while the low excitation galaxies (LEG) are a slowlyevolving or non-evolving group of radio-sources. This has also been recognised by other authors who suggest that some young radio-loud AGN can be short-lived objects on time scales $10^{4}-10^{5}$ years (Reynolds \& Begelman 1997; Aleksander 2000; Marecki et al. 2003; Czerny et al. 2009; Kunert-Bajraszewska et al. 2010, Shulevski et al. 2015). Very recently, based on the optical and X-ray observations, Schawinski et al.(2015) concluded that the whole lifetime of AGN consists of numerous short phases (each phase typically lasts for $\sim 10^{5}$ years) alternating between high and low accretion rate.

The analysis of the radio, optical and X-ray properties of a sample of low luminosity compact (LLC) sources carried out by us over the last few years provides new information in this active field of research. These studies brought new interesting clues on the evolution of radio-loud AGN (Kunert-Bajraszewska \& Labiano 2010; Kunert-Bajraszewska et al. 2010, 2014) and we shortly summarize them here. 


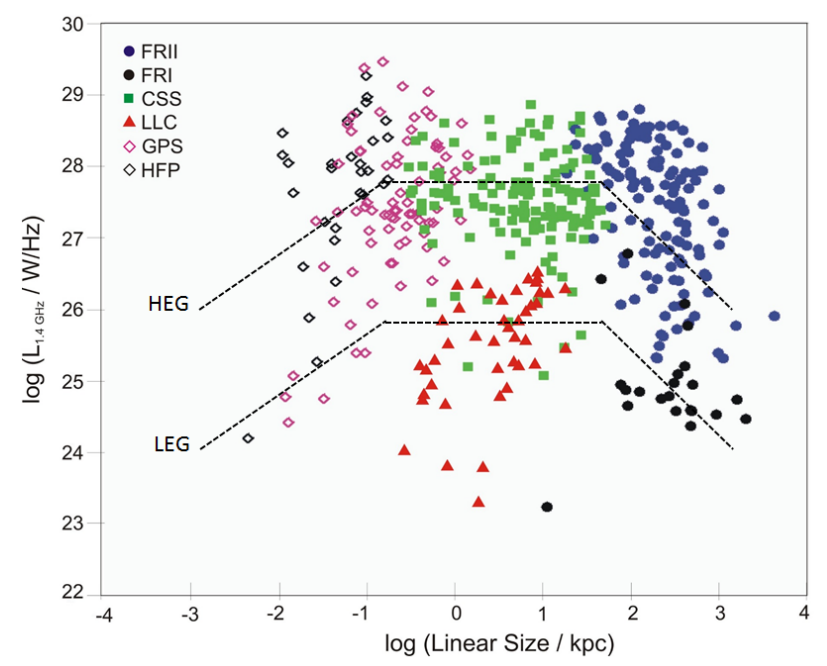

Fig. 1 Radio power - linear size diagram for radio-loud AGN. The new sample of low luminosity compact (LLC) sources are indicated with red triangles. High frequency peakers (HFP) are a sub-population of compact AGN defined by Dallacasa et al.(2000). Additionally two independent evolutionary tracks for HEG and LEG sources are outlined (see section 3.2.

\section{Our sample}

The main aim of our project was to explore the lower luminosity region of the radio power - linear size plane occupied by compact radio sources. Thus, the most important criterion for selecting the sample was the radio luminosity threshold. To look for these objects we combined information from the Faint Images of the Radio Sky at 20-cm (FIRST) (White et al. 1997), the Green Bank 6-cm (GB6) survey (Condon et al. 1994) and Sloan Digital Sky Survey (SDSS). The selection criteria we used (for details see $\mathrm{Ku}-$ nert-Bajraszewska et al. 2010) resulted in approximately onethird of the sources having a value of $1.4 \mathrm{GHz}$ radio luminosity comparable to FR Is. The final sample consisted of 44 LLC objects. They are in poorly studied to date area of the radio power - linear size plot (Figure 1). The radio observations of LLC objects were carried out with MERLIN at L-band and C-band in a few observational campaigns.

As the next step we examined the optical properties of LLC sources and applied for them the division for low and high excitation galaxies (Kunert-Bajraszewska \& Labiano 2010). The obtained results are in agreement with a dualpopulation model and evolution for large scale radio AGN (Buttiglione et al. 2010; Best \& Heckman 2012).

And finally, the new Chandra observations of some LLC objects revealed interesting individual cases (Kunert-Bajraszewska, Siemiginowska \& Labiano 2013) and helped us with further analysis of the properties and evolution of CSS sources (Kunert-Bajraszewska et al. 2014).

\section{Discussion}

\subsection{Impact of environment ...}

The majority of LLC sources were resolved with multi-element radio linked interferometer network (MERLIN) and revealed many types of radio structures. A large percentage ( $\sim 86$ per cent) of objects with a detected core show difference in the brightness of the lobes located on both its sides. The same result has been previously found for the CSS source population studied by Saikia et al.(2001). It is probably due to the fact that a young source is confined within the host galaxy and needs to struggle with the interstellar medium during the expansion (Labiano 2008; Holt, Tadhunter \& Morganti 2009; Kawakatu et al. 2009; Chandola, Gupta \& Saikia 2013; Dallacasa et al. 2013; Geréb et al. 2015). If during the evolution jets are expanding in an inhomogeneous ambient medium as an effect we observe disrupted radio structure which can be preserved to larger scale (Wagner et al. 2012). The most extreme cases of disturbed large scale structures belong to hybrid morphology radio sources (HYMORS). This is a rare class of double-lobed radio sources where each of the two lobes clearly exhibits a different FR morphology (Gopal-Krishna \& Wiita 2000; Gawroński et al. 2006). Recent high resolution radio observations of HYMORS showed that their parsec-scale structures are similar to that of FR II sources. Thus the different radio behaviour that we observe must arise on a larger, probably kiloparsec-scale (Cegłowski et al. 2013), where the jets of young AGN try to break through the ISM.

One exception to the above interpretation is the binary quasar FIRST J164311.3+315618 that we found in our sample (Kunert-Bajraszewska \& Janiuk 2011). Its complex and distorted radio and optical morphology indicates the intermittent activity with a possible rapid change of the jet direction and/or restarting of the jet due to the interaction with the companion.

Another distinctive feature of LLC sources is the fact that about $30 \%$ of them have weak extended radio emission and breaking-up structures. The weak radio core emission is also present and in some cases it can stand for a large fraction of the whole emission of the source. We estimated the core dominance parameter $\mathrm{R}=\mathrm{S}_{\text {core }} /\left(\mathrm{S}_{\text {tot }}-\mathrm{S}_{\text {core }}\right)$ for those LLC objects that have C-band observations and well separated radio core in the $5 \mathrm{GHz}$ image $(0854+210$, $0923+079,1154+435,1156+470,1506+345)$. They have on average $\left(\mathrm{R}_{\mathrm{ave}}=0.23\right)$ higher core dominance than larger FR IIs $\left(R_{\text {ave }}=0.02\right)$ and FR Is $\left(R_{\text {ave }}=0.04\right.$; Morganti, Killeen, Tadhunter 1993) and strong CSS sources $\left(\mathrm{R}_{\text {ave }}<\right.$ 0.1; Saikia et al.2001). The statistics is low but seems to be in agreement with recently reported high values of $\mathrm{R}$ for the more radio weak FR 0 sources (Ghisellini et al. 2014; Sadler et al. 2014; Baldi, Capetti, Giovannini 2015). These objects similarly to some LLC sources are not able to develop strong extended radio structures for some reason. As we discussed in Kunert-Bajraszewska et al.(2010) different mechanisms and interactions may play an important role 


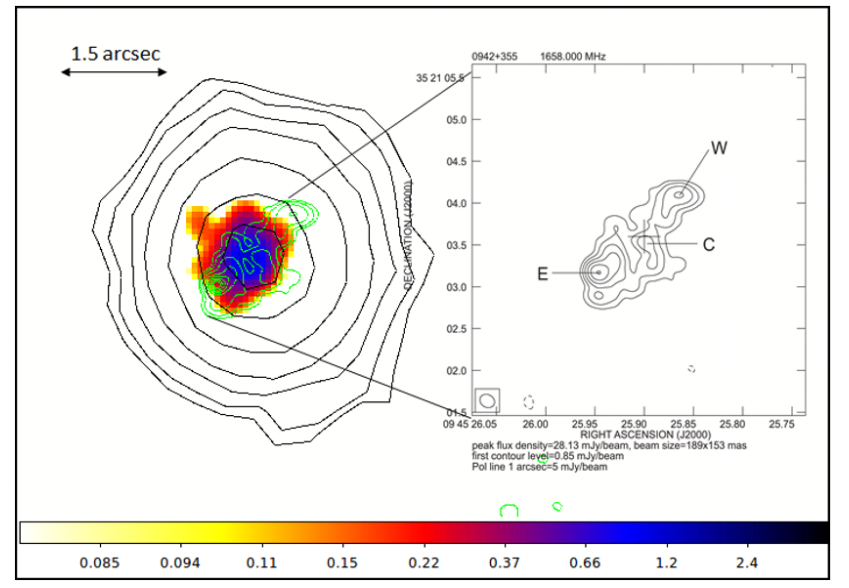

Fig. 2 SDSS optical (black contours), Chandra X-ray (color) and MERLIN $1.6 \mathrm{GHz}$ (green contours) emission from $0942+355$. Additionally the enlarged radio map is also shown. We have used 0.15 pixel blocking for the X-ray image. The cross indicates the position of an optical counterpart taken from the SDSS. The radio contours increase by factor of 2 , the first contour level corresponds to $\approx 3 \sigma$ image noise and amounts to $0.85 \mathrm{mJy} / \mathrm{beam}$.

here. This could be the already mentioned jet-ISM interactions but also differences in the properties of the so called central engine: black hole spin or accretion mode. The later can cause the production of weaker jets that are more vulnerable to instabilities and disruption. It has already been recognised by some authors (Hardcastle, Evans \& Croston 2007; Buttiglione et al. 2010; Best \& Heckman 2012) that once we take into account the optical spectrum (accretion mode) of the FR I and FR II sources we can talk about two sub-population of radio-loud AGN: the high and low excitation galaxies (HEG and LEG, respectively). We have extended these optical studies to CSS and GPS sources.

\section{2 ... or difference in the accretion mode?}

Optical spectroscopic information can play an important role in gaining a better understanding of the properties of central engines of radio sources. The emission-line diagnostic diagrams are able to distinguish H II regions ionized by young stars from gas clouds ionized by nuclear activity. Additionally, emission line luminosities show broad connection with radio power what points to a common energy source for both (Buttiglione et al. 2010; Best \& Heckman 2012).

SDSS optical data are available for most of the LLC sources and using the emission-line ratios we have classified the sources as HEGs and LEGs (Kunert-Bajraszewska $\&$ Labiano 2010). We have compared the [O III] luminosity with the radio properties for LLC sources, and expanded the sample with other CSS, GPS sources and FR I and FR II objects. The whole sample shows that the HEG sources are brighter than LEG in the [O III] line by a factor of 10 notwithstanding their linear size that is the evolutionary stage. The main evolution scenario (GPS $\rightarrow$ CSS $\rightarrow$ FR II) for radio-loud

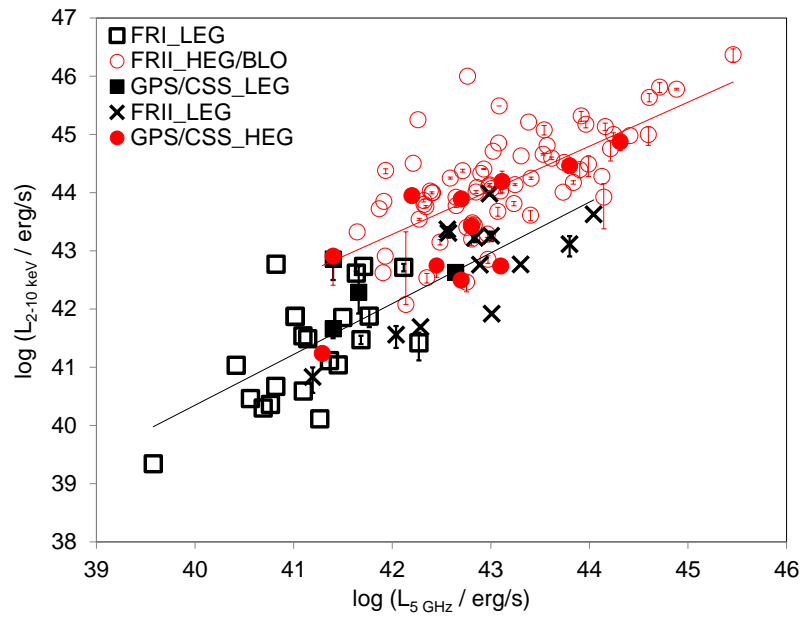

Fig. $3 \quad 2-10 \mathrm{keV}-5 \mathrm{GHz}$ luminosity diagram for AGN classified as HEGs and LEGs (reprinted from KunertBajraszewska et al.(2014)). The classification for FR I and FR II sources were taken from Buttiglione et al. 2010, where the broad line objects (BLO) are considered as members of the HEG class.

AGN was proposed years ago (Readhead et al. 1996; Fanti et al. 1995; O’Dea \& Baum 1997). However, once the optical division is included, these sources seem to evolve in parallel: $\mathrm{GPS}_{\mathrm{LEG}}-\mathrm{CSS}_{\mathrm{LEG}}-\mathrm{FR}_{\mathrm{LEG}}$ and $\mathrm{GPS}_{\mathrm{HEG}}-$ $\mathrm{CSS}_{\mathrm{HEG}}-\mathrm{FR}_{\mathrm{HEG}}$ (indicated schematically in Figure 1). Concerning LEG, it is still not clear if CSS $_{\text {LEG }}$ would evolve directly to FR I LEG or go through a FR II LEG phase before the FR I LEG.

\subsection{Radio/X-ray luminosity plane}

A pilot sample consisting of seven LLC sources was selected for X-ray observations using Chandra. Four of them have been detected, the other three have upper limit estimations for X-ray flux (Kunert-Bajraszewska et al. 2014). One of the objects, 1321+045, appeared to be associated with an X-ray cluster (Kunert-Bajraszewska, Siemiginowska \& Labiano 2013). The Chandra ACIS-S image of one of the sources with the largest number of X-ray photons is shown in Figure 2 as an example. We also overlayed the SDSS optical and radio MERLIN $1.6 \mathrm{GHz}$ contours on the X-ray emission to picture the scale of these emissions.

We again searched through the literature for other GPS and CSS sources and large scale FR Is and FR IIs with X-ray detections in order to determine the nature of the relation between morphology, X-ray properties and excitation modes in radio-loud AGN. We have compared the X-ray luminosity of the radio sources from all above mentioned groups with their radio properties. The large-scale FR II sources and strong GPS and CSS objects settle at higher X-ray and radio luminosities, while the low power CSSs occupy the space among FR I objects that are weaker in X-rays.

Once the HEG/LEG classification is applied also to the $\mathrm{X}$-ray detected AGN, both these groups occupy distinct lo- 
cus in the radio/X-ray luminosity plane as shown in Figure 3.

And finally we have tested the AGN evolution models by comparing the radio/X-ray luminosity ratio with the size of the sources, and indirectly, with their age. We found that the less radio powerful FR Is have higher radio/X-ray luminosity ratio than many FR IIs what may imply higher $\mathrm{X}$-ray luminosity decrease with radio power in FR Is than FR IIs. This is in agreement with previously postulated different X-rays emission origins. In the case of FR Is the Xray emission is connected with the base of the jet while in FR IIs it originates from accretion (Siemiginowska et al. 2008, Tengstrand et al. 2009). The same can be true for compact sources as well. The results suggest that below certain level of radio luminosity GPSs and CSSs start to resemble FR Is.

\section{Summary}

The analysis of the radio and X-ray properties of LLC sources together with their spectroscopic features indicate that most of them (especially the HEGs) will evolve finally to FR IIs. However, they are the tip of the iceberg and much larger population of short-lived low power radio sources are still wating to be explored with high sensitivity radio surveys. They probably belong to the branch of slowly evolving low excitation galaxies and could be the missing precursors of large-scale FR I sources.

Acknowledgements. The research leading to these results has received funding from the European Commission Seventh Framework Programme (FP/2007-2013) under grant agreement No 283393 (RadioNet3)

This research has made use of the SDSS. Funding for SDSSIII has been provided by the Alfred P. Sloan Foundation, the Participating Institutions, the National Science Foundation, and the U.S. Department of Energy Office of Science. The SDSS-III web site is http://www.sdss3.org/

This research has made use of data obtained by the Chandra Xray Observatory, and Chandra X-ray Center (CXC) in the application packages CIAO, ChIPS, and Sherpa. This research is funded in part by NASA contract NAS8-39073. Partial support for this work was provided by the Chandra grants GO1-12124X.

\section{References}

Aleksander, P.: 2000, MNRAS, 319, 8

An, T., Baan, W. A.: 2012, ApJ, 760, 77

Baldi, R. D., Capetti, A., Giovannini, G.: 2015, A\&A, 576, 38

Best, F. N., Heckman, T. M.: 2012, MNRAS, 421, 1569

Buttiglione S., Capetti, A., Celotti, A., Axon, D.J., Chiaberge, M., Macchetto, F.D., Sparks, W.B.: 2010, A\&A, 509, 6

Cegłowski, M., Gawroński, M. P., Kunert-Bajraszewska, M.: 2013, A\&A, 557, 75

Chandola, Y., Gupta, N., Saikia, D. J.: 2013, MNRAS, 429, 2380

Condon J.J., Broderick J.J., Seielstad G.A., Douglas K., Gregory P.C.: 1994, AJ, 107, 1829

Czerny, B., Siemiginowska, A., Janiuk, A., Nikiel-Wroczyński, B., Stawarz, Ł.: 2009, ApJ, 698, 840
Dallacasa, D., Stanghellini, C., Centoza, M., Fanti, R.: 2000, A\&A, 363, 887

Dallacasa, D., Orienti, M., Fanti, C., Fanti, R., Stanghellini, C.: 2013, MNRAS, 433, 147

Fanaroff, B. L., \& Riley, J. M.: 1974, MNRAS 167, 31

Fanti, C., Fanti, R., Dallacasa, D., et al.: 1995, A\&A 302, 317

Gawroński, M. P., Marecki, A., Kunert-Bajraszewska, M., Kus, A. J.: 2006, A\&A, 447, 63

Geréb, K., Maccagni, F. M., Morganti, R., Oosterloo, T. A.: 2015, A\&A, 575, 44

Ghisellini, G., Tavecchio, F., Maraschi, L., Celotti, A., Sbarrato, T.: 2014, Nature, 515, 376

Gopal-Krishna; Wiita, P. J.: 2000, A\&A, 363, 507

Hardcastle, M. J., Evans, D. A., Croston, J. H.: 2007, MNRAS, 376,1849

Holt, J., Tadhunter, C. N., Morganti, R.: 2009, MNRAS, 400, 589

Kawakatu, N, Kino, M., Nagai, H.: 2009, ApJ, 697, 173L

Kunert-Bajraszewska, M. \& Thomasson, P.: 2009, AN, 330, 210

Kunert-Bajraszewska, M.,Gawroński, M. P., Labiano, A., Siemiginowska, A.: 2010, MNRAS, 408, 2261

Kunert-Bajraszewska, M., Labiano, A.: 2010, MNRAS, 408, 2279

Kunert-Bajraszewska, M., Janiuk, A.: 2011, ApJ, 736, 125

Kunert-Bajraszewska, M., Siemiginowska, A., Labiano, A.: 2013, ApJ, 772, L7

Kunert-Bajraszewska, M., Labiano, A., Siemiginowska, A., Guainazzi, M.: 2014, MNRAS, 437, 3063

Labiano, A.: 2008, A\&A, 488, 59

Maccagni, F. M., Morganti, R., Oosterloo, T. A., Mahony, E. K.: 2014, A\&A, 571, 67

Maciel, T., Aleksander, P.: 2014, MNRAS, 442, 3469

Marecki, A., Spencer, R. E., \& Kunert, M.: 2003, PASA 20, 46

Morganti, R., Killeen, N. E. B., Tadhunter, C. N.: 1993, MNRAS, 263, 1023

O’Dea, C. P., \& Baum, S. A.: 1997, AJ 113, 148

Perucho, M., Quilis, V., Martí, J.M.: 2011, ApJ, 743, 4

Readhead, A. C. S., Taylor, G. B., Xu, W., et al.: 1996, ApJ 460, 612

Reynolds, C. S., \& Begelman, M. C.: 1997, ApJ, 487, L135

Sadler, E. M., Ekers, R. D., Mahony, E. K., Mauch, T., Murphy, T.: 2014, MNRAS, 438, 796

Saikia, D. J., Jeyakumar, S., Salter, C. J., et al.: 2001, MNRAS 321,37

Schawinski, K., Koss, M., Berney, S., Sartori, L.: 2015, MNRAS, 451,2517

Shulevski, A., Morganti, R., Barthel, P.D. et al.: 2015, A\&A, 579, 27

Siemiginowska, A., LaMassa, S., Aldcroft, T. L., Bechtold, Jill; Elvis, M.: 2008, ApJ, 684, 811

Snellen, I. A. G., Schilizzi, R. T., Miley, G. K., de Bruyn, A. G., Bremer, M. N., Röttgering, H. J. A.: 2000, MNRAS, 319, 445

Tengstrand, O., Guainazzi, M., Siemiginowska, A., Fonseca Bonilla, N., Labiano, A., Worrall, D. M., Grandi, P., Piconcelli, E.: 2009, A\&A, 501, 89

Turner, R. J., Shabala, S. S.: 2015, ApJ, 806, 59

Wagner, A. Y., Bicknell, G. V., Umemura, M.: 2012, ApJ, 757, 136

White, R. L., Becker, R. H., Helfand, D. J., \& Gregg, M. D.: 1997, ApJ 475, 479 